\title{
Red Wine Polyphenol Compounds Favor Neovascularisation through Estrogen Receptor $\alpha$ - Independent Mechanism in Mice
}

\author{
Matthieu Chalopin ${ }^{\top 1}$, Raffaella Soleti ${ }^{\top 1}$, Tarek Benameur ${ }^{1}$, Angela Tesse ${ }^{1}$, Sébastien Faure ${ }^{1}$, Maria \\ Carmen Martínez', Ramaroson Andriantsitohaina ${ }^{1,2 *}$
}

1 INSERM U1063, Stress Oxydant et Pathologies Métaboliques, Angers, France, 2 Centre Hospitalier Universitaire d'Angers, Angers, France

\begin{abstract}
Red wine polyphenol compounds (RWPC) exert paradoxical effects depending on the dose on post-ischemic neovascularisation. Low dose RWPC $(0.2 \mathrm{mg} / \mathrm{kg} / \mathrm{day})$ is pro-angiogenic, whereas high dose $(20 \mathrm{mg} / \mathrm{kg} / \mathrm{day})$ is antiangiogenic. We recently reported that the endothelial effect of RWPC is mediated through the activation of a redoxsensitive pathway, mitochondrial biogenesis and the activation of $\alpha$ isoform of the estrogen receptor $(E R \alpha)$. Here, we investigated the implication of ER $\alpha$ on angiogenic properties of RWPC. Using ovariectomized mice lacking ER $\alpha$ treated with high dose of RWPC after hindlimb ischemia, we examined blood flow reperfusion, vascular density, nitric oxide (NO) production, expression and activation of proteins involved in angiogenic process and muscle energy sensing network. As expected, high dose of RWPC treatment reduced both blood flow and vascular density in muscles of mice expressing ER $\alpha$. These effects were associated with reduced NO production resulting from diminished activity of eNOS. In the absence of RWPC, ER $\alpha$ deficient mice showed a reduced neo-vascularisation associated with a decreased NO production. Surprisingly in mice lacking $E R \alpha$, high dose of RWPC increased blood flow and capillary density in conjunction with increased NO pathway and production as well as VEGF expression. Of particular interest is the activation of Sirt-1, AMPK $\alpha$ and PGC-1 $\alpha / \beta$ axis in ischemic hindlimb from both strains. Altogether, the results highlight a pro-angiogenic property of RWPC via an ER $\alpha$ independent mechanism that is associated with an up-regulation of energy sensing network. This study brings a corner stone of a novel pathway for RWPC to correct cardiovascular diseases associated with failed neovascularisation.
\end{abstract}

Citation: Chalopin M, Soleti R, Benameur T, Tesse A, Faure S, et al. (2014) Red Wine Polyphenol Compounds Favor Neovascularisation through Estrogen Receptor $\alpha$-Independent Mechanism in Mice. PLoS ONE 9(10): e110080. doi:10.1371/journal.pone.0110080

Editor: Jane-Lise Samuel, Inserm, France

Received April 29, 2014; Accepted September 15, 2014; Published October 9, 2014

Copyright: (C) 2014 Chalopin et al. This is an open-access article distributed under the terms of the Creative Commons Attribution License, which permits unrestricted use, distribution, and reproduction in any medium, provided the original author and source are credited.

Data Availability: The authors confirm that all data underlying the findings are fully available without restriction. All relevant data are within the paper.

Funding: This work was supported by institutional grants from INSERM and Université d'Angers. RA was supported by a Contrat d'Interface INSERM. MC is the recipient of a doctoral fellowship from French Education Ministry. The funders had no role in study design, data collection and analysis, decision to publish, or preparation of the manuscript.

Competing Interests: The authors have declared that no competing interests exist.

* Email: ramaroson.andriantsitohaina@univ-angers.fr

- MC and RS are first co-authors on this work.

\section{Introduction}

Angiogenesis, the sprouting of new blood vessel, is crucial for all tissue growth, expansion and repair [1]. Angiogenesis is a multistep process, starting with endothelial cell activation, vasodilatation and increased vascular permeability, followed by endothelial cell migration and proliferation into the perivascular space. Finally, endothelial cells assemble to form a new vessel [2]. This process occurs in physiological states as embryogenesis or after injury as well in pathological issues, such as cancer growth [3], rheumatoid arthritis [4] or diabetic retinopathy [5].

Numerous epidemiological studies have consolidated the idea that a moderate consumption of red wine is associated with a reduced cardiovascular risk [6]. The beneficial effects of red wine are reported due to its polyphenol components (RWPG). We have recently reported that the alpha isoform of the estrogen receptor $(\mathrm{ER} \alpha)$ is one of the targets involved in vascular effects of RWPG [7]. Both RWPC, and delphinidin, an anthocyanin that possesses the same pharmacological profile than the total extract, interact directly with activator site of $\mathrm{ER} \alpha$ inducing the activation of ERK/Src/eNOS pathway leading to endothelial NO release and then, vasodilation [7].

RWPC are reported to possess anti-angiogenic properties. In a swine study of chronic ischemia, high dose of resveratrol (100 mg/ $\mathrm{kg}$ /day) inhibited new vessel formation, probably through an increased expression of angiostatin and thrombospondin leading to decreased expression of VE-cadherin and other pro-angiogenic factors [8]. In a rat model of peripheral ischemia, we have found that low doses $(0.06 \mathrm{mg} / \mathrm{kg} /$ day $)$ of delphinidin have no effect on blood flow recovery, however higher doses $(0.6 \mathrm{mg} / \mathrm{kg} /$ day $)$ have anti-angiogenic effects as evidenced by impaired blood flow reperfusion and decreased vascular density in the ischemic leg [9]. In an in vivo model of avascular rabbit cornea, quercetin $(100 \mu \mathrm{M})$ inhibited VEGF-induced endothelial cell functions and angiogenesis and prevented VEGF-induced ERK1/2 phosphorylation [10]. On the other hand, RWPC protect against deleterious effects of cardiac and cerebral ischemia [11,12], the correction of which requires pro-angiogenic properties to produce new blood 
vessels in order to rescue the infarcted area. These dual effects of RWPG remain unexplained. We have highlighted a dosedependent effect of polyphenols in an in vivo model of angiogenesis triggered by peripheral ischemia to explain this paradoxical effect of RWPC [9]. A low dose of RWPC promoted angiogenesis by activation of NO, Akt/PI3K and p38 MAPK pathways, and increasing VEGF expression without altering either MMP activity or NF- $\mathrm{NB}$ expression. In contrast, a high dose of RWPC reduced angiogenesis via an inhibition of both NO/VEGF and Akt/PI3K pathways, whereas MMP activity was reduced in association with phospho-p38 and NF- $\mathrm{BB}$ expression [9].

Beside, RWPC activate Sirtuin 1 (Sirt-1), 5' adenosine monophosphate-activated protein kinase $(\mathrm{AMPK} \alpha)$ and peroxisome proliferator-activated receptor gamma coactivator-1 (PGG$1 \alpha)$ which are implicated in energy expenses and angiogenesis [1317]. The mechanisms involved in angiogenic effects of RWPC have already been described in a number of studies, but the molecular target is still not fully understood. This study was designed to assess the role played by $\mathrm{ER} \alpha$ in paradoxical angiogenic effects of RWPC. Using ovariectomized genetically modified mice lacking $\mathrm{ER} \alpha$ fed chronically with high dose of RWPC, we first studied blood flow recovery after surgical hindlimb ischemia. Then, we evaluated underlying angiogenic mechanisms in hindlimb muscles, through analysis of vascular density, NO production, as well as expression and activation of proteins involved in angiogenic process and muscle energy sensing network.

\section{Methods and Materials}

\section{Products}

RWPG was obtained from Société Française des Distilleries (Vallon Pont d'Arc, France). The composition is, in $\mathrm{mg} / \mathrm{g}$ of dry powder: 480 proanthocyanidins, 61 total anthocyanins, 19 free anthocyanins, 38 catechin, 18 hydroxycinnamic acids, 14 flavonols and 370 polymeric tannins.

For animal feeding, RWPC was dissolved in water and given by daily gavage.

\section{Ethics Statement}

The procedures followed in the care and euthanasia of the study animals was in accordance with the European Community standards on the care and use of laboratory animal and was approved by the Ethical Committee for Animal Research of Angers University. The animals were housed in a regulated environment with a constant ambient temperature of $24^{\circ} \mathrm{C}$. They had free access to standard laboratory food and water.

\section{Animals}

Twelve-week-old female ER $\alpha$ Wild Type (WT) or Knock Out (KO) mice were ovariectomized. After 7 days, mice were treated for 28 days with RWPG ( $20 \mathrm{mg} / \mathrm{kg} /$ day) or water $(\mathrm{n}=5$ animals for each group). One day after beginning of treatment, mice were anesthetised with isoflurane and underwent surgery to induce unilateral hindlimb ischemia. The ligature was performed on the left femoral artery proximal to the bifurcation to the saphenous and popliteal arteries, as previously described [9,18]. After 7, 14, 21 and 28 days of ligature, blood flow was measured as described below. At 28 day, mice were euthanized and tissues were sampled for biochemical and histological analysis.

\section{Quantification of perfusion: Laser-Doppler Blood Flow (LDBF) analysis}

In order to provide a functional evidence of ischemia, laser doppler perfusion imaging was performed in anesthetized mice, as previously described [18]. Animals were settled on a heating plate to maintain a stable cutaneous temperature in order to minimize temperature variation throughout the experiments. Leg perfusion was then measured using a Laser Doppler flow probe (PF 408, Perimed, Stockholm, Sweden). Blood flow was recorded during $\sim 3$ min. At least 2 flow measurements were performed per leg. Blood flow perfusion was expressed as a ratio of left (ischemic) to right (non-ischemic) leg, as described by Limbourg et al. [19].

\section{Vascular density}

Vascular density, as an index of neovascularisation, was examined by counting the number of vessels taken from the ischemic and non-ischemic limbs. Ischemic and non-ischemic gastrocnemius muscles were dissected and embedded in TissueTek O.C.T (Sakura Finetek, Zoeterwoude, The Netherlands). Cryosections $(7 \mu \mathrm{m})$ were fixed $\left(5 \mathrm{~min}\right.$ at $\left.-20^{\circ} \mathrm{C}\right)$ in $100 \%$ methanol, and saturated ( $1 \mathrm{~h}$ at room temperature) in blocking buffer (5\% non fat dry milk in PBS and $0.05 \%$ Tween 20). Fixed and blocked tissue sections were incubated overnight at $4{ }^{\circ} \mathrm{C}$ with rat anti-mouse CD31 antibody (1:100, BD Biosciences, San Jose, CA). After three washes, tissue sections were incubated (1 h at room temperature) with goat anti-rat IgG fluorescein-conjugated (1:100, Southern Biotech, Birmingham, AL) to identify vessels as described by Limbourg et al. [19]. After final washes, sections were mounted on glass slides. MRC-1024ES confocal equipment mounted on a Nikon Eclipse TE 300 inverted microscope was used for the optical sectioning of the tissue. Digital image recording was performed using the Laser Sharp Software. Vessels were quantified using Image J software and counted in at least four randomly selected fields for each muscle section, and the mean value for each section was calculated (magnification $\mathrm{x} 40$ ).

\section{NO spin trapping and electron paramagnetic resonance (EPR) studies}

Detection of NO production was performed using the technique with $\mathrm{Fe}^{2+}$ diethyldithiocarbamate (DETC, Sigma-Aldrich) as spin trap. Briefly, after treatment, aortas, ischemic or non-ischemic gastrocnemius and soleus muscles were dissected for NO production measurement by their incubation for $30 \mathrm{~min}$ in Krebs-Hepes buffer containing: BSA $(20.5 \mathrm{~g} / \mathrm{L}), \mathrm{CaCl}_{2}(3 \mathrm{mM})$ and L-Arginine $(0.8 \mathrm{mM})$. NaDETC $(3.6 \mathrm{mg})$ and $\mathrm{FeSO}_{4}-7 \mathrm{H}_{2} \mathrm{O}$ (2.25 mg, Sigma-Aldrich) were separately dissolved under nitrogen gas bubbling in $10 \mathrm{ml}$ volumes of ice-cold Krebs-Hepes buffer. The solutions were rapidly mixed to obtain a pale yellow-brown opalescent colloid Fe(DETC $)_{2}$ solution $(0.4 \mathrm{mM})$, which was used immediately to incubate organs for $45 \mathrm{~min}$ at $37^{\circ} \mathrm{C}$. After incubation spin trap was removed, organs were immersed in physiological salt solution and frozen in liquid nitrogen.

NO measurement was performed on a table-top x-band spectrometer Miniscope (Magnettech, MS200, Berlin, Germany). Recordings were made at $-196^{\circ} \mathrm{C}$, using a Dewar flask. Instrument settings were $10 \mathrm{~mW}$ of microwave power, $1 \mathrm{mT}$ of amplitude modulation, $100 \mathrm{kHz}$ of modulation frequency, $150 \mathrm{~s}$ of sweep time and 5 scans. Signals were quantified by measuring the total amplitude, after correction of baseline as done previously [20]. 


\section{Western Blotting}

After 28 days of treatment, mice were sacrificed and gastrocnemius and soleus muscles from ischemic and non ischemic hindlimb were cut out and were homogenized and lysed. Proteins $(80 \mu \mathrm{g})$ were separated on $10 \%$ SDS-PAGE electrophoresis gel. Blots were probed with eNOS, phospho-eNOS Ser 1177, phospho-eNOS Thr 495, cav-1, phospho-cav-1, NF-кB, VEGF, Sirt-1, PGC- $1 \alpha / \beta$ and AMPK $\alpha$ antibodies (1:500, Cell Signalling, Danvers, MA). A polyclonal anti-mouse $\beta$-actin antibody (1:2000, Sigma-Aldrich) was used to visualize protein gel loading. Bound antibodies were detected with a secondary peroxidase-conjugated anti-rabbit or anti-mouse IgG (Promega, Charbonnieres, France). The blots were visualized using the enhanced chemiluminescence system (ECL Plus, Amersham Biosciences, Piscatawat, NJ) or for phosphorylated proteins using the Super Signal West Femto (Thermo Scientific Pierce, Brebières, France) and quantified by densitometry and normalized to $\beta$-actin expression. Results are expressed with ischemic/non ischemic ratio for each protein.

\section{Data analysis}

Data are represented as mean \pm SEM, $\mathrm{n}$ represents the number of experiments. Statistical analyses were performed by a one way analysis of variance (ANOVA), and Mann-Whitney U tests or ANOVA for repeated measures and subsequent Bonferroni post hoc test. $\mathrm{P}<0.05$ was considered to be statistically significant.

\section{Results}

Laser doppler quantification of perfusion in the hindlimb

Evaluation of hindlimb blood flow perfusion was performed weekly in both ischemic and non-ischemic leg, by laser doppler blood flow analysis. Ischemia was induced by ligature and excision of the left femoral artery, as previously described [18]. No difference could be observed in the degree of post-operative ischemia between the four groups of mice. Seven days after ligature, foot blood flow was significantly lower in the ischemic than in the non-ischemic leg, but no differences were found in blood flow recovery between the four groups (Fig. 1a). Fourteen and 21 days after ligature, only $\mathrm{ER} \alpha \mathrm{KO}$ mice treated with RWPC displayed higher blood flow recovery in comparison with other groups (Fig. 1a). At day 28 after ligature in WT mice, RWPC decreased by 0.57 -fold the ischemic/non-ischemic blood flow ratio $(\mathrm{P}<0.01)$ compared with control. Deletion of $\mathrm{ER} \alpha$ leaded to a reduced perfusion by 0.7 -fold the ischemic/non-ischemic blood flow ratio ws control $(\mathrm{P}<0.05)$. Surprisingly in $\mathrm{KO}$ mice, RWPC induced a greater blood flow perfusion compared to untreated $\mathrm{KO}$ mice (Fig. la-c). This increase in blood flow recovery was also greater than that obtained in WT mice $(\mathrm{P}<0.01)$ with or without RWPC treatment $(\mathrm{P}<0.05)$.

\section{Vascular density}

Data from the laser doppler analysis were confirmed by vascular density measurement using a fluorescent CD31 staining visualized by confocal microscopy. Twenty eight days after ligature, RWPC decreased the vascular density expressed as ischemic/normal ratio by 0.58 -fold $(\mathrm{P}<0.001)$ compared with the control WT mice. In mice lacking $\mathrm{ER} \alpha$, vascular density was reduced by 0.42 -fold $(\mathrm{P}<$ 0.001) in comparison with WT mice. Interestingly, RWPG treatment in $\mathrm{KO}$ mice induced an increase in vascular density compared to WT mice $(1.18$-fold; $\mathrm{P}<0.01)$ as well as WT treated mice $(2.02$-fold; $\mathrm{P}<0.001)$ and control $\mathrm{KO}$ mice $(2.82$-fold; $\mathrm{P}<$ 0.001) (Fig. 2).

Altogether, we report that the anti-angiogenic ability of RWPC requires the presence of $\mathrm{ER} \alpha$. It is also found a pro-angiogenic activity of RWPC via a mechanism independent of the presence of $\mathrm{ER} \alpha$.

\section{NO production in aorta and hindlimb muscle}

NO production was assessed both in the aorta as a control regarding a non-ischemic tissue (Fig. 3a) and in the ischemic skeletal muscle (Fig. 3b). As expected, RWPG significantly reduced NO production in the aorta from WT mice $(0.7$-fold; $\mathrm{P}<0.05)$. Also, deletion of $\mathrm{ER} \alpha$ decreased the NO production in aorta (0.7-fold; $\mathrm{P}<0.05)$. Interestingly, RWPC significantly enhanced NO production in aorta from $\mathrm{ER} \alpha$ deficient mice compared either from control WT $(0.7$-fold; $\mathrm{P}<0.01)$, RWPGtreated WT mice $(1.9$-fold; $\mathrm{P}<0.001)$ or control KO $(1.9$-fold: $\mathrm{P}<$ $0.001)$.

In skeletal muscle from WT mice, RWPG decreased significantly NO production compared to control (2.6-fold; $\mathrm{P}<0.001$ ). Mice lacking of $\mathrm{ER} \alpha$ displayed a NO level significant lower than WT (1.7-fold; $\mathrm{P}<0.05)$. Surprisingly, RWPG significantly increased NO production in $\mathrm{ER} \alpha$ deficient mice compared either to control WT (1.7-fold; P<0.01), RWPC-treated WT mice (4.5-fold; $\mathrm{P}<0.001)$ or control KO (2.9-fold; $\mathrm{P}<0.01)$. These data suggest that NO level of hindlimb ischemia was correlated to the changes in blood flow in all groups of mice studied.

\section{Effects of ER $\alpha$ deletion and RWPC in non ischemic muscles}

The impact of $\mathrm{ER} \alpha$ deficiency and RWPG on signalling pathways in non-ischemic hindlimb muscles has been evaluated. As shown in Figure 4, among the proteins evaluated, deletion of $\mathrm{ER} \alpha$ decreased only phosphorylation of cav-1 in comparison with WT mice normalized to $\beta$-actin expression. In the present study, we also found that RWPC decreased Sirt-1/AMPK $\alpha /$ PGC- $1 \alpha / \beta$ expressions in the non ischemic area in both WT and KO mice. It might be possible that in vivo ischemic conditions are need for RWPC to activate these pathways, and such conditions are not fulfilled in the non ischemic area.

\section{Effect of RWPC on proteins in ischemic/non-ischemic skeletal muscle ratio: role of $\mathrm{ER} \alpha$}

As shown in Figure 5 (a, b), in skeletal muscles from WT mice, although RWPC did not modify eNOS expression, they decreased eNOS activity as revealed by a reduced phosphorylation on its activator site (Ser1177) without changes in phosphorylation on its inhibitor site (Thr495). Also, RWPC decreased cav-1 phosphorylation without affecting its expression. All these modifications are consistent with observed decreased NO production. In muscles taken from $\mathrm{ER} \alpha$ deficient mice, increased eNOS expression was associated with enhanced phosphorylation on Thr495 and decreased cav-1 expression and phosphorylation, leading to decreased activity and reduced NO production. In mice lacking $\mathrm{ER} \alpha$, treatment with RWPC induced an increased activity of eNOS due to its phosphorylation on Serl177 associated with decreased cav-1 expression and activation, which is in agreement with the detected NO level in this tissue. Whereas RWPC were not able to significantly modify expression of VEGF and NF- $\kappa \mathrm{B}$ in WT mice, RWPG increased expression of both proteins in KO mice. RWPG treatment enhanced expression of AMPK $\alpha$ and PGC- $1 \alpha / \beta$ in tissues taken from WT mice. Deletion of ER $\alpha$ was associated with a decreased expression of, AMPK $\alpha$ and PGC- $1 \alpha$ / $\beta$, in hindlimb muscles when compared to WT mice. However, RWPG treatment enhanced expression of Sirt-1, AMPK $\alpha$ and PGC- $1 \alpha / \beta$ in tissues taken from $\mathrm{ER} \alpha$ deficient mice in a similar extent than WT mice. 


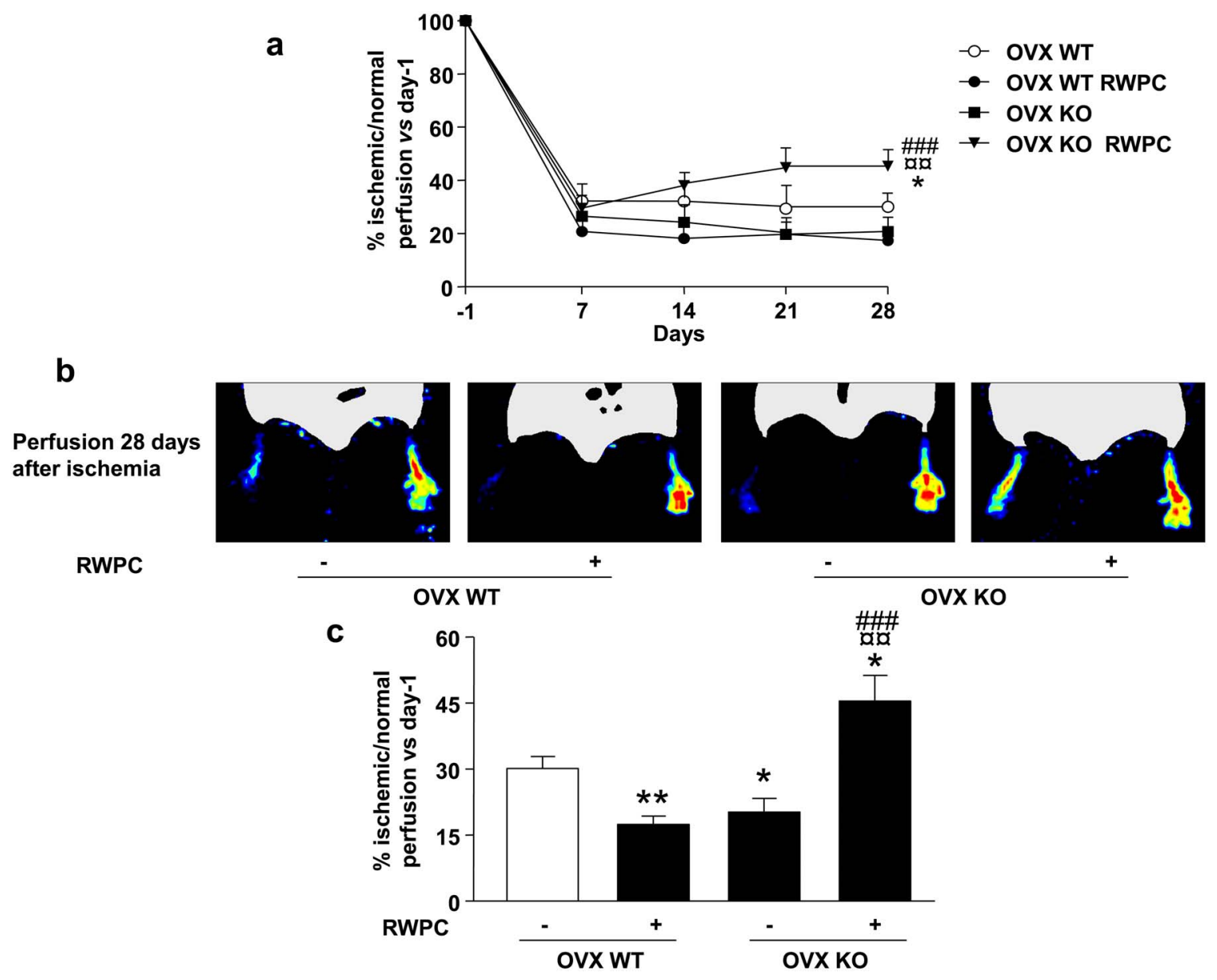

Figure 1. Evaluation of leg neovascularisation after femoral artery ligature in ovariectomized (OVX) ERa WT and KO mice treated or not with $20 \mathrm{mg} / \mathbf{k g} / \mathrm{day}$ of red wine polyphenol compounds (RWPC). (a) Quantification of blood perfusion at different times (one day before ligature (day-1), and days 7, 14, 21 and 28 after ligature) in four groups of mice ( $n=5 /$ group). Values are expressed in mean \pm SEM as the ischemic/ non ischemic leg ratio vs day-1. (b) Blood flow perfusion ( $n=5 /$ group) with typical images. (c) Quantification of perfusion. Values are expressed in mean \pm SEM as the ischemic/non ischemic leg ratio vs day-1. ${ }^{*} P<0.05,{ }^{*} P<0.01$ vs control OVX WT; aaP $<0.01$ vs OVX WT treated; \#\#\#P<0.001 vs control OVX KO.

doi:10.1371/journal.pone.0110080.g001

These data suggest that RWPC exerted their anti-angiogenic property in presence of $\mathrm{ER} \alpha$ by decreasing NO production. More interesting, we describe for the first time that the pro-angiogenic effect of RWPG resulting from activation of eNOS is associated with increased Sirt-1, AMPK $\alpha$ and PGC- $1 \alpha / \beta$ pathways independently from $\mathrm{ER} \alpha$.

\section{Discussion}

The present study shows that, after peripheral ischemia, deletion of $\mathrm{ER} \alpha$ leads to reduced neovascularisation associated with a decreased of NO production, diminution of eNOS activity, and AMPK $\alpha$ and PGG- $1 \alpha / \beta$ expressions. In addition, the described results confirm that high dose of RWPG reduces neovascularisation in mice subjected to hindlimb ischemia via inhibition of NO pathway despite their ability to increase Sirt-1, $\mathrm{AMPK} \alpha$ and PGC- $1 \alpha / \beta$ expressions. Interestingly, they also demonstrate that deletion of $\mathrm{ER} \alpha$ unmasks pro-angiogenic activities of RWPG in association with increased NO/VEGF pathways and NF- $\kappa \mathrm{B}$ without affecting their ability to activate Sirt$1 / \mathrm{AMPK} \alpha / \mathrm{PGC}-1 \alpha / \beta$ axis. Thus, NO/VEGF, NF- $\kappa \mathrm{B}$ and Sirt$1 / \mathrm{AMPK} \alpha / \mathrm{PGC}-1 \alpha / \beta$ axis plays a role in the pro-angiogenic effect of RWPG via an ER $\alpha$-independent mechanism.
We have previously shown that in the rat model of induced peripheral ischemia, the same dose of RWPG used in the present study, had anti-angiogenic properties via inhibition of $\mathrm{NO}$ pathway and NF- $\kappa \mathrm{B}$ expression [9]. Similar effects are observed in the present study in WT mice, confirming that reduced angiogenesis induced by high doses of RWPG involves of NO/ VEGF and Akt/PI3K pathways and reduction of MMP activity, phosphorylation of p38 and NF- $\kappa \mathrm{B}$ expression. In agreement with these results other authors have shown that a higher dose of RWPC (100 mg/kg/day) prevents the expression of pro-angiogenic factors [21], including VEGF and eNOS most likely by inhibiting oxidative stress. As previously reported, the molecular identity of the compounds responsible for the anti-angiogenic properties of high dose of RWPG is not fully known. However, delphinidin one the major compounds found in RWPG, inhibited angiogenesis in a rat model of hindlimb ischemia [9]. Thus, this compound displays the same pharmacological profile than the total extract. Indeed, our group has shown that delphinidin inhibits in vitro and in vivo angiogenesis. In human umbilical vein endothelial cells the anti-proliferative properties of delphinidin involves cyclin D1- and A-dependent pathway [22]. Furthermore, in chicken embryo chorioallantoic membrane assay, delphinidin is 

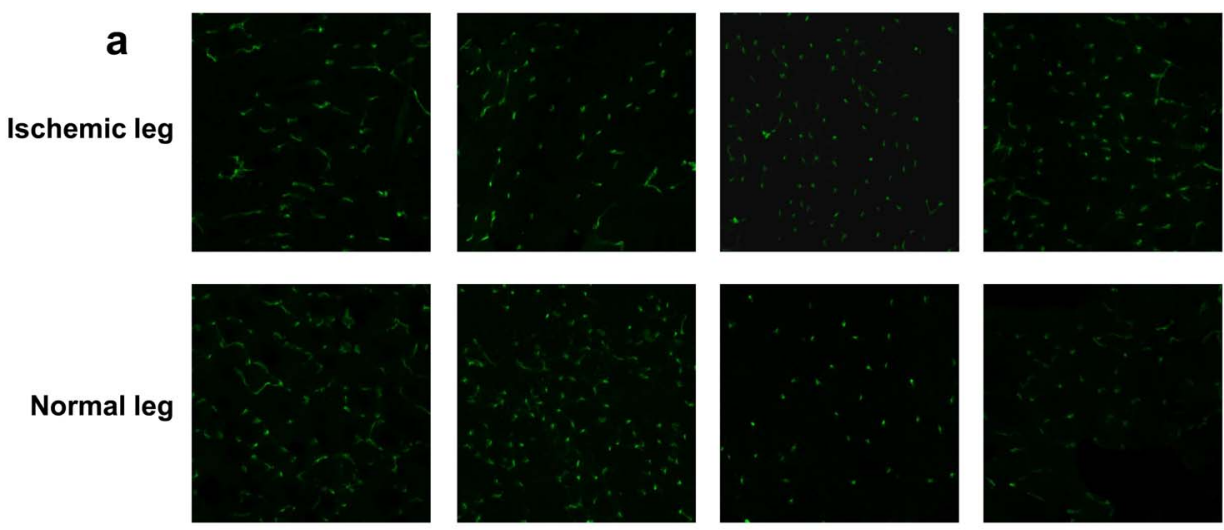

RWPC
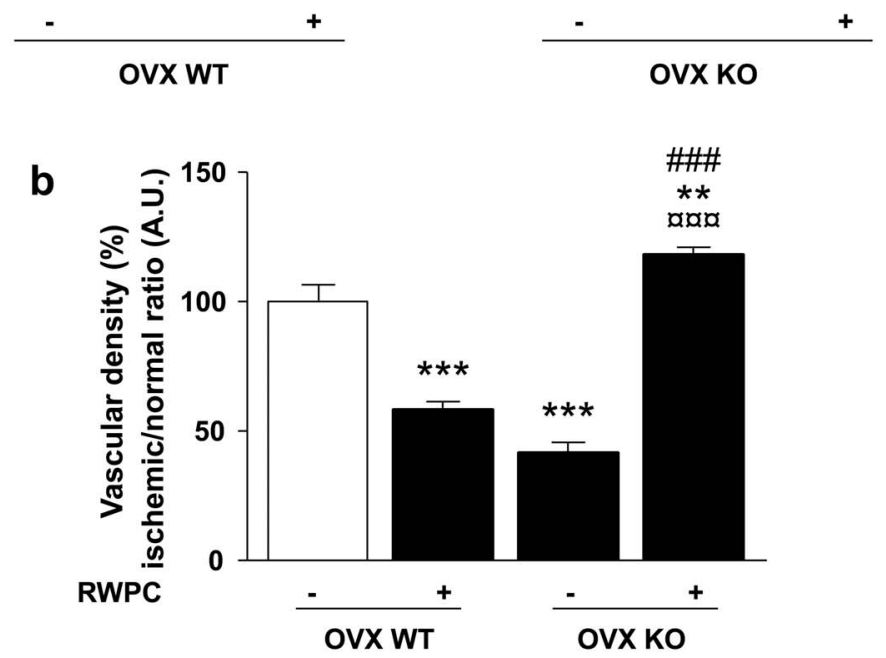

Figure 2. Evaluation of capillary density in ovariectomized (OVX) ERa WT and KO mice treated or not with $20 \mathrm{mg} / \mathrm{kg} / \mathrm{day}$ of RWPC for 28 days. (a) Typical images of capillary density by CD31 staining in ischemic and normal legs. (b) Quantification of capillary density. Values are expressed in mean \pm SEM as the ischemic/non ischemic leg ratio $(\mathrm{n}=5)$. ${ }^{* *} P<0.01,{ }^{* * *} P<0.001$ vs. control OVX WT; axaP $<0.001$ vs OVX WT treated; $\# \# \# P<0.001$ vs control OVX KO.

doi:10.1371/journal.pone.0110080.g002

able to decrease capillary development through an ERK1/2 phosphorylation, independent of NO pathway and correlated with suppression of cell progression by blocking the cell cycle in G0/G1 phase in bovine aortic endothelial cells [23]. Altogether, further studies are needed to sort out the molecular identities of RWPC for its anti-angiogenic effect that might explain some of the differential mechanisms found compared to delphinidin.

\section{a}

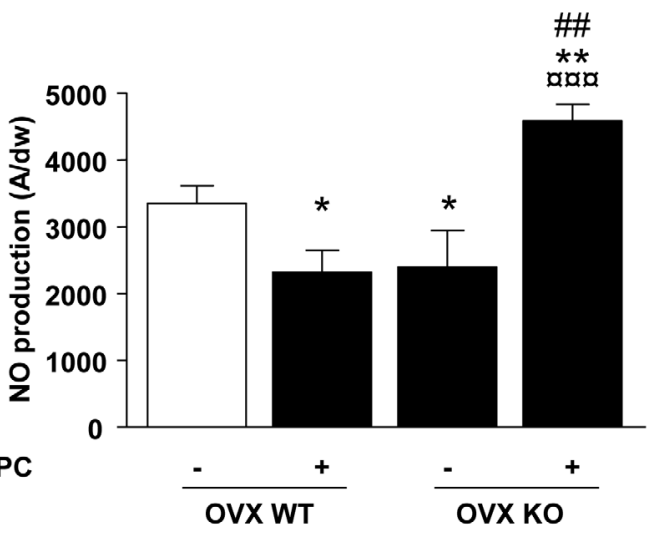

b

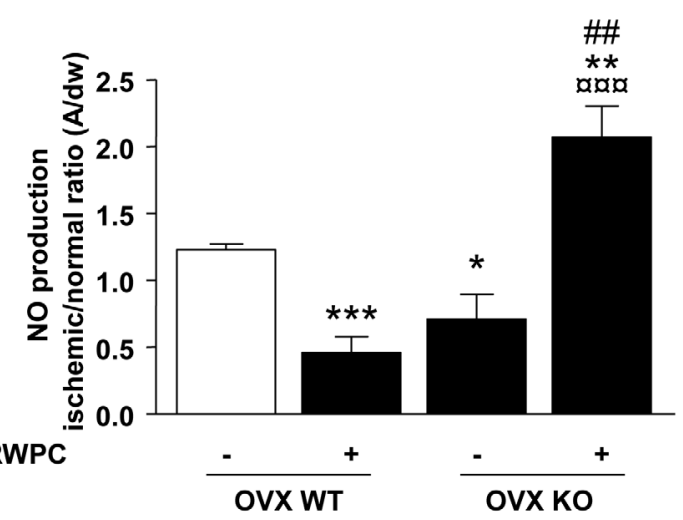

Figure 3. Quantification of the amplitude of NO signals in aorta (a) and in hindlimb muscles (b) from ovariectomized (OVX) ERa WT and KO mice treated or not with $\mathbf{2 0} \mathbf{~ m g / k g / d a y ~ o f ~ R W P C ~ f o r ~} \mathbf{2 8}$ days. Values are expressed as amplitude/mg of dried weight (dw) of aorta or as ratio of amplitude/mg of $d w$ of muscle (mean \pm SEM) $(n=5)$. ${ }^{*} P<0.05$, ${ }^{* *} P<0.01$, ${ }^{* *} P<0.001$ vs. control group; axa $P<0.001$ vs OVX WT treated; $\# \# \# P<0.001$ vs control OVX KO.

doi:10.1371/journal.pone.0110080.g003 


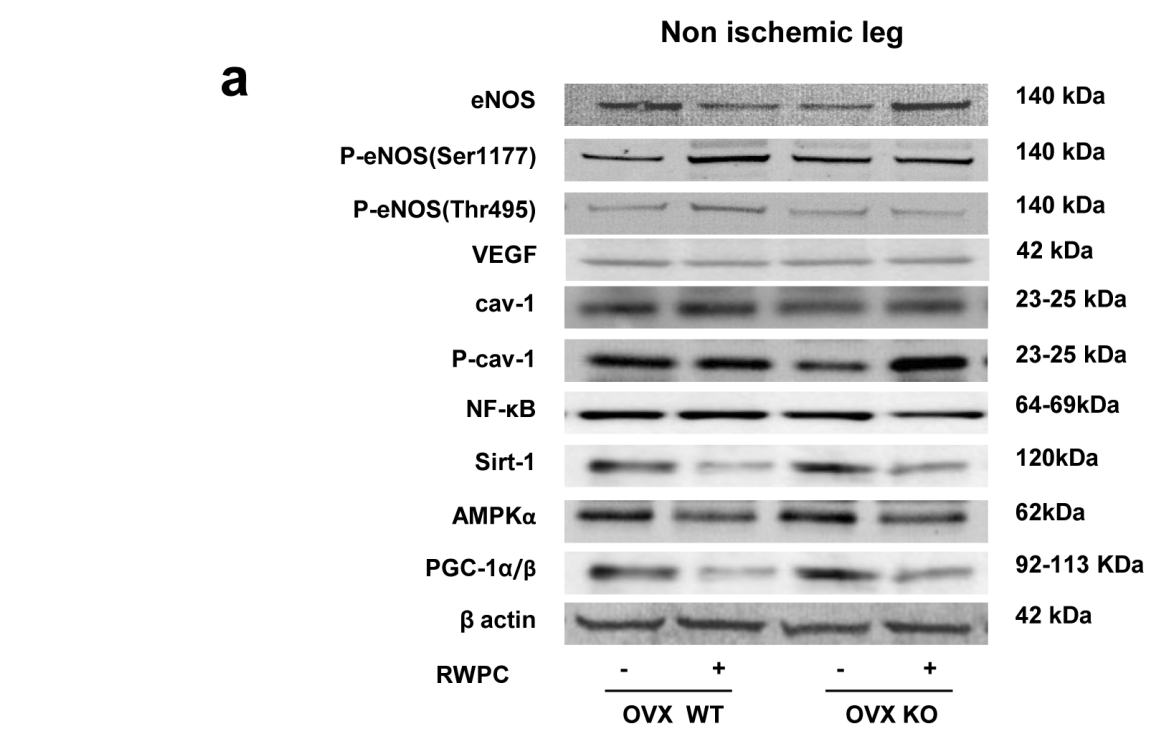

b
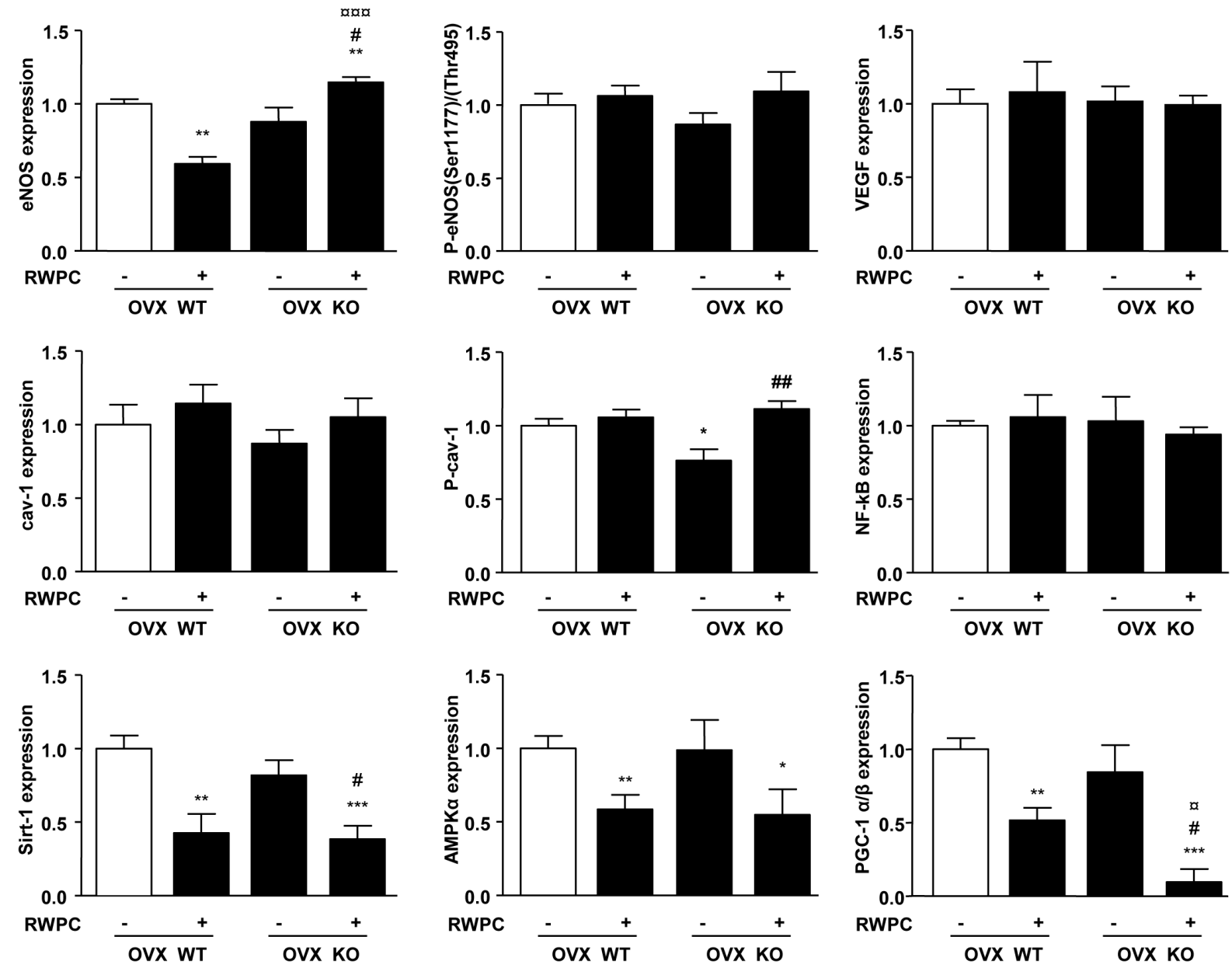

Figure 4. Evaluation of the protein expression levels extracted from the right (non ischemic) gastrocnemius and soleus muscles in ovarectomised (OVX) ER $\alpha$ WT and KO mice treated or not with $\mathbf{2 0} \mathbf{~ m g / k g}$ of RWPC for $\mathbf{2 8}$ days. Representative images of blots showing protein expression levels in non ischemic leg muscle (a) Quantification of protein expressions expressed normalized to $\beta$-actin expression, (b) (mean

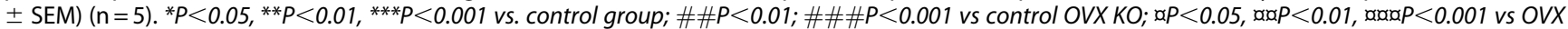
WT treated.

doi:10.1371/journal.pone.0110080.g004 
Ischemic leg

a

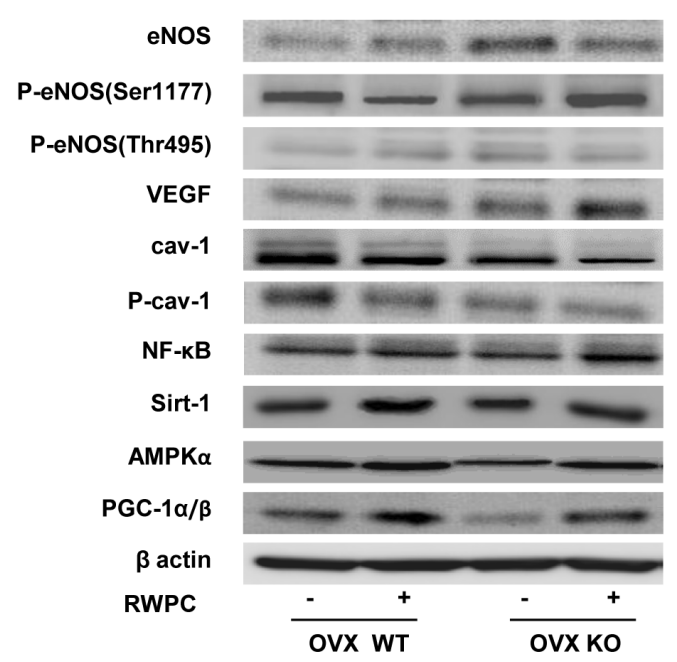

140 kDa

140 kDa

140 kDa

$42 \mathrm{kDa}$

23-25 kDa

23-25 kDa

64-69kDa

$120 \mathrm{kDa}$

62kDa

92-113 KDa

$42 \mathrm{kDa}$

b
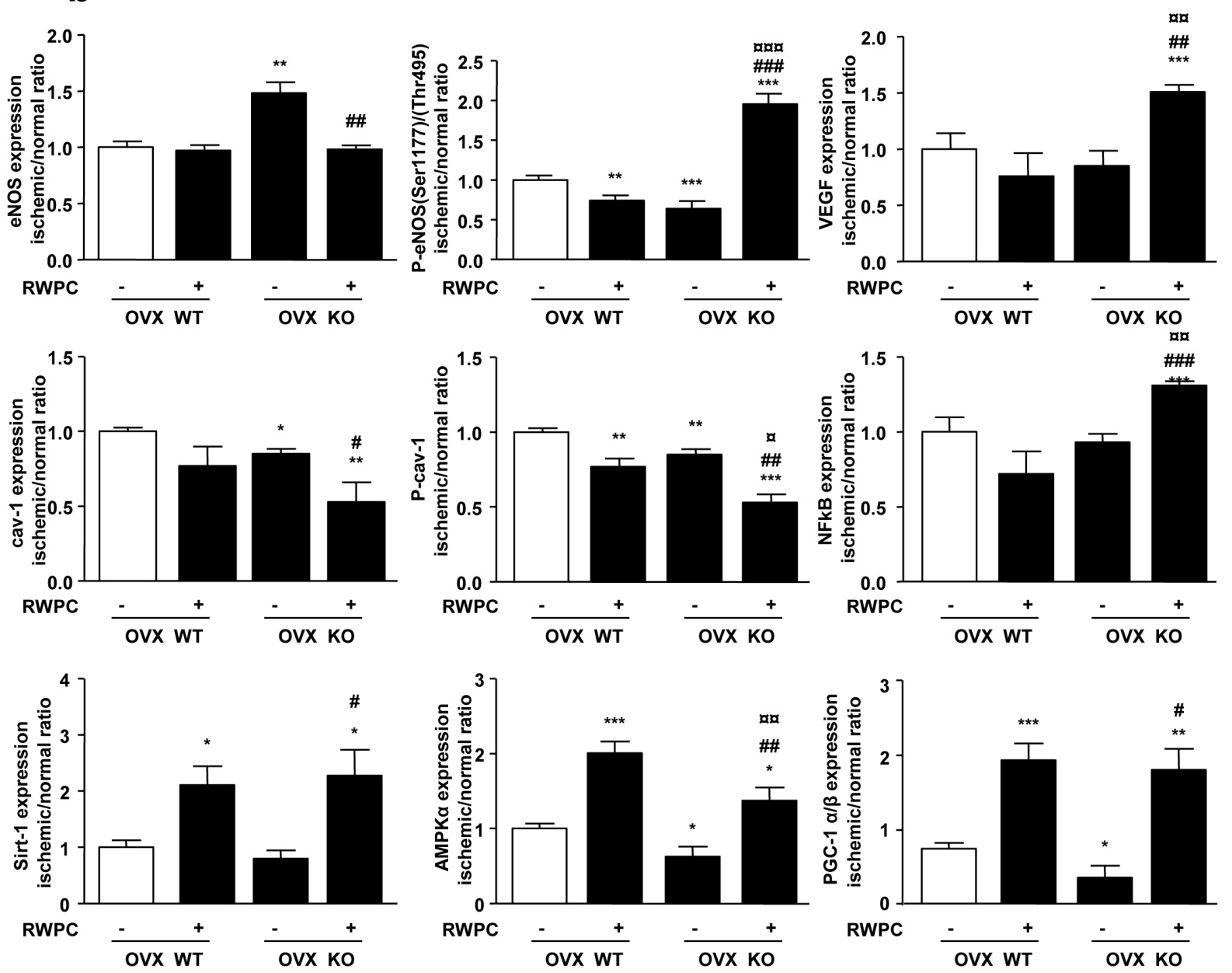

Figure 5. Evaluation of the protein expression levels extracted from the right (normal) and left (ischemic) gastrocnemius and soleus muscle in ovarectomised (OVX) ERa WT and KO mice treated or not with $\mathbf{2 0} \mathbf{~ m g / k g}$ of RWPC for 28 days. Representative images of blots showing protein expression levels in ischemic leg muscle (a) Quantification of protein expressions expressed as a ratio of normal/ischemic protein expression in comparison with WT control group normalized to $\beta$-actin expression, (b) (mean $\pm \mathrm{SEM})(\mathrm{n}=5)$. ${ }^{*} P<0.05,{ }^{* *} P<0.01,{ }^{* * *} P<0.001$ vs.

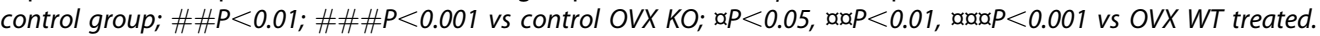

doi:10.1371/journal.pone.0110080.g005 


\section{High dose RWPC (20mg/kg/day)}

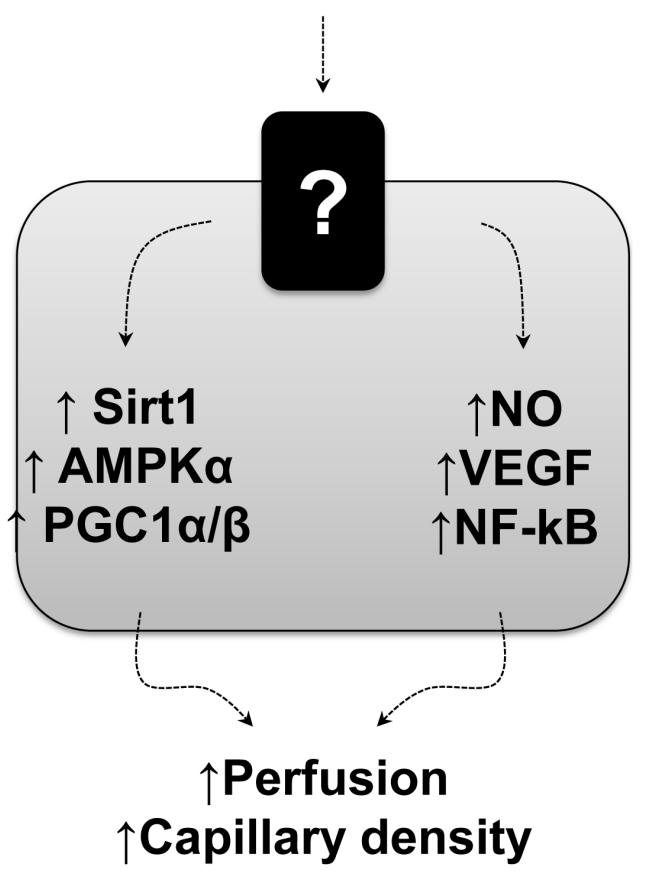

\section{Pro-angiogenic effect}

Figure 6. Scheme of pro angiogenic effect of RWPC. Deletion of $\mathrm{ER} \alpha$ unmask the ability of RWPC to enhance neovascularisation VEGF/ NO/NF-kB and Sirt-1/AMPK $\alpha / P G C-1 \alpha / \beta$ pathways. These effects concur to increased blood perfusion and capillary density. doi:10.1371/journal.pone.0110080.g006

It has been shown that high $\left(20 \mathrm{mg} / \mathrm{kg}\right.$ in vivo, $10^{-2} \mathrm{~g} / \mathrm{l}$ in vitro) or low $\left(0.2 \mathrm{mg} / \mathrm{kg}\right.$ in vivo, $10^{-4} \mathrm{~g} / \mathrm{l}$ in vitro) $\mathrm{RWPC}$ increase endothelial NO production [7,20,24-27] via a NADPH oxidase sensitive pathway and the activation of the ER $\alpha$. Very recently, we found that low concentration of RWPC induces in vitro angiogenesis by a mechanism sensitive to ER inhibitor involving both NADH oxydase and NO pathways associated with increased mitochondrial capacity [27]. Also, $\mathrm{ER} \alpha$ agonists promote angiogenesis in human myometrial microvascular endothelial cells through the activation of $\mathrm{NO}$ and VEGF pathways [28]. Estrogens also affect positively angiogenesis by inducing mobilization and recruitment of endothelial progenitor cells [28]. In the present study, deletion of $\mathrm{ER} \alpha$ decreased $\mathrm{NO}$ production via reduced eNOS activity and reduced AMPK $\alpha$ and PGC- $1 \alpha / \beta$ expressions. In conjunction with this study, Jesmin et al. [29] have previously reported disrupted levels of VEGF associated with decreased capillary density in $\mathrm{ER} \alpha \mathrm{KO}$ mice. Altogether we reinforce the notion that $\mathrm{ER} \alpha$ plays a role in neovascularisation after peripheral ischemia. As previously indicated, the identification of molecular compounds which elicit the effects reported in the present study has not been carried out. Nevertheless, it was found that low and high doses of RWPC are adequate to produce a sufficient circulating concentration of compounds able to modulate angiogenesis [9]. The latter study support the hypothesis of different doses of RWPG modulate the balance between circulating pro- and anti-angiogenic compounds that might determine the final effect of RWPG in vivo.

In addition to the effects of RWPG mentioned above in WT mice, we highlight a pro-angiogenic activity of high dose of these compounds in mice lacking $\mathrm{ER} \alpha$. These properties were associated with increased NO production through the induction of $\mathrm{NO}$ and VEGF pathways. Interestingly, high dose of RWPG enhanced Sirt- 1, AMPK $\alpha$ and PGG- $1 \alpha / \beta$ expressions in hindlimb ischemia of both wild type and $\mathrm{ER} \alpha$-deficient mice but paradoxically reduced and favoured neovascularisation in WT and $\mathrm{KO}$ mice, respectively. Sirt- 1, AMPK $\alpha$ and PGC- $1 \alpha / \beta$ have been reported to play a role in angiogenesis. Sirt-1, a class III histone deacetylase, is involved in multiple physiological processes [30] and is activated under stress condition to regulate cell cycles [31]. Interestingly, Sirt-1 is able to upregulate eNOS, reduces smooth muscle cells senescence and suppresses ROS and inflammation in arteries [32]. Sirt-1 activation by laminar flow and statin treatment also increases eNOS activity and NO production [33,34]. Thus, the interaction between Sirt-1 and eNOS might contribute to the proangiogenic properties of RWPC. Indeed, Sirt-1 is involved in angiogenic process, as mice displaying endothelial cell-specific deletion of SIRT1 present an impaired angiogenesis in response to ischemia due to its ability to deacetylate forkhead transcription factor Foxol, an essential negative regulator of blood vessel development, to restrain its anti-angiogenic activity [35]. Sirt-1 may inhibit angiogenesis by suppressing hypoxia inducible factor 1 alpha activity [36]. AMPK $\alpha$ is implicated for angiogenesis process in endothelial cells from human umbilical vein [37]. AMPK $\alpha$ can also mediate VEGF-induced NO production in human aortic endothelial cells [38]. Finally, mice lacking PGC-1 $\alpha$ fails to recover blood flow after ischemia [39] and are associated with a decreased capillary density and VEGF expression [40]. Interaction between these three molecular targets of RWPC has been reported in the literature [41]. Altogether, one can advance the hypothesis, that the ER $\alpha$-independent pathway activated by RWPC is associated to the activation of Sirt-1/AMPK $\alpha / \mathrm{PGC}-1 \alpha / \beta$ axis leading to an increased NO production and therefore participate to enhance neovascularisation under the experimental condition used. Therefore, some of RWPC are pro-angiogenic being able to activate Sirt-1/AMPK $\alpha /$ PGC- $1 \alpha / \beta$ cascade. However, the presence of $\mathrm{ER} \alpha$ hinders this effect by acting on VEGF/NO axis as

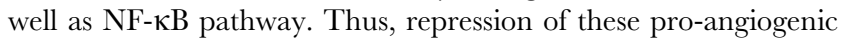
pathways became predominant and conduced led to failed angiogenesis at high dose of RWPG.

In conclusion, the present study highlights the dual effects of RWPG on neovascularisation (Fig. 6). RWPC exert their antiangiogenic activity, at least in part, via an ER $\alpha$-dependent mechanism. Of particular interest is the demonstration of increased neovascularisation due to a synergistic activation of ER $\alpha$-dependent VEGF/NO pathway and Sirt-1/AMPK $\alpha /$ PGG$1 \alpha / \beta \mathrm{ER} \alpha$-independent mechanism. Thus, this study brings a corner stone of a novel pathway for RWPG to correct cardiovascular diseases associated with default of neovascularisation.

\section{Acknowledgments}

We thank Pr. P. Chambon and Dr. A. Krust for providing mice strain and M. Wertheimer and SCAHU staff (Université d'Angers) for taking care of animals.

\section{Author Contributions}

Conceived and designed the experiments: RA. Performed the experiments: MC RS AT TB SF. Analyzed the data: MC RS AT. Contributed 
reagents/materials/analysis tools: MC RS MCM RA. Contributed to the writing of the manuscript: MC RS MCM RA.

\section{References}

1. Folkman J (1995) Seminars in Medicine of the Beth Israel Hospital, Boston. Clinical applications of research on angiogenesis. N Engl J Med 333: 17571763.

2. Bergers G, Benjamin LE (2003) Tumorigenesis and the angiogenic switch. Nat Rev Cancer 3: 401-410.

3. Folkman J (1971) Tumor angiogenesis: therapeutic implications. N Engl J Med 285: 1182-1186.

4. Szekanecz Z, Besenyei T, Paragh G, Koch AE (2009) Angiogenesis in rheumatoid arthritis. Autoimmunity 42: 563-573.

5. Crawford TN, Alfaro DV, Kerrison JB, Jablon EP (2009) Diabetic retinopathy and angiogenesis. Curr Diabetes Rev 5: 8-13.

6. Renaud S, de Lorgeril M (1992) Wine, alcohol, platelets, and the French paradox for coronary heart disease. Lancet 339: 1523-1526.

7. Chalopin M, Tesse A, Martínez MC, Rognan D, Arnal J, et al. (2010) Estrogen receptor alpha as a key target of red wine polyphenols action on the endothelium. PLoS ONE 5: e8554.

8. Robich MP, Chu LM, Chaudray M, Nezafat R, Han Y, et al. (2010) Antiangiogenic effect of high-dose resveratrol in a swine model of metabolic syndrome. Surgery 148: 453-462.

9. Baron-Menguy C, Bocquet A, Guihot A, Chappard D, Amiot M, et al. (2007) Effects of red wine polyphenols on postischemic neovascularization model in rats: low doses are proangiogenic, high doses anti-angiogenic. FASEB J. 21: 3511-3521.

10. Donnini S, Finetti F, Lusini L, Morbidelli L, Cheynier V, et al. (2006) Divergent effects of quercetin conjugates on angiogenesis. B J Nutr 95: 1016-1023.

11. Ralay Ranaivo H, Diebolt M, Andriantsitohaina R (2004) Wine polyphenols induce hypotension, and decrease cardiac reactivity and infarct size in rats: involvement of nitric oxide. Br J Pharmacol 142: 671-678.

12. Ritz MF, Ratajczak P, Curin Y, Cam E, Mendelowitsch A, et al. (2008) Chronic treatment with red wine polyphenol compounds mediates neuroprotection in a rat model of ischemic cerebral stroke. J Nutr 138: 519-525.

13. Mattagajasingh I, Kim CS, Naqvi A, Yamamori T, Hoffman TA, et al. (2007) SIRT1 promotes endothelium dependent vascular relaxation by activating endothelial nitric oxide synthase. Proc Natl Acad Sci USA 104: 14855-14860.

14. Csiszar A, Labinskyy N, Pinto JT, Ballabh P, Zhang H, et al. (2009) Resveratrol induces mitochondrial biogenesis in endothelial cells. Am J Physiol Heart Circ Physiol 297: H13-H20.

15. Raval AP, Dave KR, Pérez-Pinzón MA (2006) Resveratrol mimics ischemic preconditioning in the brain. J Cereb Blood Flow Metab 26: 1141-1147.

16. Zang M, Xu S, Maitland-Toolan KA, Zuccollo A, Hou X, et al. (2006) Polyphenols stimulate AMP-activated protein kinase, lower lipids, and inhibit accelerated atherosclerosis in diabetic LDL receptor-deficient mice. Diabetes 55: 2180-2191.

17. Howitz KT, Bitterman KJ, Cohen HY, Lamming DW, Lavu S, et al. (2003) Small molecule activators of sirtuins extend Saccharomyces cerevisiae lifespan. Nature 425: 191-196.

18. Couffinhal T, Silver M, Zheng LP, Kearney M, Witzenbichler B, et al. (1998) Mouse model of angiogenesis. Am J Pathol 152: 1667-1679.

19. Limbourg A, Korff T, Napp LC, Schaper W, Drexler H, et al. (2009) Evaluation of postnatal arteriogenesis and angiogenesis in a mouse model of hind-limb ischemia. Nat Protoc 4: 1737-1746.

20. Agouni A, Lagrue-Lak-Hal A, Mostefai HA, Tesse A, Mulder P, et al. (2009) Red wine polyphenols prevent metabolic and cardiovascular alterations associated with obesity in Zucker fatty rats $(\mathrm{Fa} / \mathrm{Fa})$. PLoS ONE 4: e5557.

21. Walter A, Etienne-Selloum N, Brasse D, Khallouf H, Bronner C, et al. (2010) Intake of grape-derived polyphenols reduces C26 tumor growth by inhibiting angiogenesis and inducing apoptosis. FASEB J 24: 3360-3369.
22. Martin S, Favot L, Matz R, Lugnier C, Andriantsitohaina R (2003) Delphinidin inhibits endothelial cell proliferation and cell cycle progression through a transient activation of ERK-1/-2. Biochem Pharmacol 65: 669-675.

23. Favot L, Martin S, Keravis T, Andriantsitohaina R, Lugnier C (2003) Involvement of cyclin-dependent pathway in the inhibitory effect of delphinidin on angiogenesis. Cardiovasc Res 59: 479-487.

24. Martin S, Andriambeloson E, Takeda K, Andriantsitohaina R (2002) Red wine polyphenols increase calcium in bovine aortic endothelial cells: a basis to elucidate signalling pathways leading to nitric oxide production. $\mathrm{Br} \mathrm{J}$ Pharmacol 135: 1579-1587.

25. Andriambeloson E, Magnier C, Haan-Archipoff G, Lobstein A, Anton R, et al. (1998) Natural dietary polyphenolic compounds cause endothelium-dependent vasorelaxation in rat thoracic aorta. J Nutr 128: 2324-2333.

26. Andriambeloson E, Kleschyov AL, Muller B, Beretz A, Stoclet JC, et al. (1997) Nitric oxide production and endothelium-dependent vasorelaxation induced by wine polyphenols in rat aorta. Br J Pharmacol 120: 1053-1058.

27. Duluc L, Jacques G, Soleti R, Iacobazzi F, Simard G, et al. (2013) Modulation of mitochondrial capacity and angiogenesis by red wine polyphenols via estrogen receptor, NADPH oxidase and nitric oxide synthase pathways. Int J Biochem Cell Biol 45: 783-791.

28. Losordo DW, Isner JM (2001) Estrogen and angiogenesis: A review. Arterioscler Thromb Vasc Biol 21: 6-12.

29. Jesmin S, Mowa GN, Sultana SN, Shimojo N, Togashi H, et al. (2010) VEGF signaling is disrupted in the hearts of mice lacking estrogen receptor alpha. Eur J Pharmacol 641: 168-178.

30. Haigis MC, Sinclair DA (2010) Mammalian sirtuins: biological insights and disease relevance. Annu Rev Pathol 5: 253-295.

31. Brunet A, Sweeney LB, Sturgill JF, Chua KF, Greer PL, et al. (2004) Stressdependent regulation of FOXO transcription factors by the SIRT1 deacetylase. Science 303: 2011-2015.

32. Brandes RP (2004) Activating SIRT1: a new strategy to prevent atherosclerosis? Cardiovasc Res 80: 163-164.

33. Chen Z, Peng IC, Cui X, Li YS, Chien S, et al. (2010) Shear stress, SIRT1, and vascular homeostasis. Proc Natl Acad Sci U SA 107: 10268-10273.

34. Ota H, Eto M, Kano MR, Kahyo T, Setou M, et al. (2010) Induction of endothelial nitric oxide synthase, SIRT1, and catalase by statins inhibits endothelial senescence through the Akt pathway. Arterioscler Thromb Vasc Biol 30: 2205-2211.

35. Potente M, Ghaeni L, Baldessari D, Mostoslavsky R, Rossig, et al. (2007) SIRT1 controls endothelial angiogenic functions during vascular growth. Genes Dev. 21: $2644-58$.

36. Lim JH, Lee YM, Chun YS, Chen J, Kim JE, et al. (2010) Sirtuin 1 modulates cellular responses to hypoxia by deacetylating hypoxia-inducible factor lalpha. Mol Cell 38: 864-878.

37. Nagata D, Mogi M, Walsh K (2003) AMP-activated protein kinase (AMPK) signaling in endothelial cells is essential for angiogenesis in response to hypoxic stress. J Biol Chem 278: 31000-31006.

38. Reihill JA, Ewart M, Hardie DG, Salt IP (2007) AMP-activated protein kinase mediates VEGF-stimulated endothelial NO production. Biochem Biophys Res Commun 354: 1084-1088.

39. Arany Z, Foo S, Ma Y, Ruas JL, Bommi-Reddy A, et al. (2008) HIFindependent regulation of VEGF and angiogenesis by the transcriptional coactivator PGC-1alpha. Nature 451: 1008-1012.

40. Leick L, Hellsten Y, Fentz J, Lyngby SS, Wojtaszewski JFP, et al. (2009) PGClalpha mediates exercise-induced skeletal muscle VEGF expression in mice. Am J Physiol Endocrinol Metab 97: E92-103.

41. Andriantsitohaina R, Auger C, Chataigneau T, Étienne-Selloum N, Li H, et al. (2012) Molecular mechanisms of the cardiovascular protective effects of polyphenols. Br J Nutr 108: 1532-1549. 